\title{
Potential energy surface and reactive collisions for the $\mathrm{Au}+\mathrm{H}_{2}$ system
}

\author{
Alexander Zanchet, ${ }^{1}$ Octavio Roncero, ${ }^{1, a)}$ Salama Omar, ${ }^{2}$ Miguel Paniagua, ${ }^{3}$ and \\ Alfredo Aguado $^{3}$ \\ ${ }^{1}$ Unidad Asociada UAM-CSIC, Instituto de Fíisica Fundamental, C.S.I.C. Serrano 123, \\ Madrid 28006, Spain \\ ${ }^{2}$ Departamento de Química Física, Facultad de Ciencias C-XIV, Universidad Autónoma de Madrid, \\ Madrid 28049, Spain \\ ${ }^{3}$ Unidad Asociada UAM-CSIC, Departamento de Química Física, Facultad de Ciencias C-XIV, \\ Universidad Autónoma de Madrid, Madrid 28049, Spain
}

(Received 17 November 2009; accepted 17 December 2009; published online 15 January 2010)

\begin{abstract}
A global potential energy surface is obtained for the ground state of the endoergic $\operatorname{Au}\left({ }^{2} S\right)$ $+\mathrm{H}_{2}\left(\mathrm{X}^{1} \Sigma_{g}^{+}\right) \rightarrow \operatorname{AuH}\left({ }^{1} \Sigma^{+}\right)+\mathrm{H}\left({ }^{2} S\right)$ reaction. The global potential is obtained by fitting highly correlated ab initio calculations on the system, using relativistic pseudopotential for the gold atom. Several electronic states are calculated correlating with $\mathrm{Au}\left({ }^{2} S\right)+\mathrm{H}_{2}, \mathrm{Au}\left({ }^{2} D\right)+\mathrm{H}_{2}$, and $\mathrm{H}_{2}$, $\mathrm{Au}\left({ }^{2} \mathrm{P}\right)+\mathrm{H}_{2}$ asymptotes. These states show several conical intersections and curve crossings along the minimum energy reaction path which are analyzed in detail. One of them gives rise to an insertion well in which there are important contributions from the $\mathrm{Au}^{2}\left({ }^{2} D\right)$ and $\mathrm{Au}\left({ }^{2} P\right)$ states of gold, which is interesting because it is analog to the deep chemisorption well appearing in larger gold clusters. Quantum wave packet and quasiclassical trajectory dynamical calculations performed for the reaction at zero total angular momentum are in good agreement, provided that a Gaussian binning method is used to account for the zero-point energy of products. Finally, integral and differential cross sections are calculated for the reaction with quasiclassical trajectories. Two different reaction mechanisms are found, one direct and the second indirect, in which the Au atom inserts in between the two hydrogen atoms because of the existence of the insertion well discussed above. (C) 2010 American Institute of Physics. [doi:10.1063/1.3290950]
\end{abstract}

\section{INTRODUCTION}

Since the discovery of gold nanoparticles as good catalysts, ${ }^{1,2}$ an intense work has been focused on the reactivity of neutral and charged gold clusters, isolated as well as supported on different substrates. ${ }^{3}$ Reactivity is intimately connected to the electronic structure and geometry of the cluster, and gold nanoparticles present very interesting ones, ${ }^{4}$ such as nanowires, ${ }^{5-7}$ planar [two-dimensional (2D)] clusters, ${ }^{8-14}$ and cages, ${ }^{15-17}$ sometimes analog to fullerenes. ${ }^{18}$ Such peculiarity is attributed to important relativistic effects, which makes that $6 s$ and $5 d$ become closer in energy than in other coinage atoms, allowing a $s d$ hybridization, ${ }^{9}$ which produces stabilization and makes possible planar $^{19,20}$ and even spherical aromatic structures. ${ }^{15}$

The high catalytic activity of gold nanostructures is attributed typically to low coordinated atoms appearing at the edges of clusters ${ }^{21-23}$ and corrugated metal surfaces. ${ }^{24}$ In gold clusters with low number of atoms, however, this is not always the case, and it has been found, for example, that the reaction site for $\mathrm{H}_{2}$ dissociation on some special model gold cluster structures is the most correlated gold atom. ${ }^{25}$ Also, planar gold clusters are not reactive when attacked from the top, in close similitude to what happens with metal surfaces. However, reactivity may appear when planar clusters are folded $^{25}$ or attacked in their plane depending on the number of gold atoms, charge, etc. ${ }^{26}$

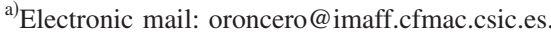

The study of the structure and reactivity of these clusters is usually performed using density functional theory (DFT) calculations, since the high number of electrons does not allow more accurate $a b$ initio methods. Different functionals typically provide different relative stability of the isomers, yielding different cluster sizes at which the 2D-threedimensional (3D) transition occurs. The application of the more highly correlated coupled cluster method, with single and double excitations and perturbative triple excitations $[\mathrm{CCSD}(\mathrm{T})]$, performed on gold clusters ${ }^{27}$ indicate that $\mathrm{Au}_{6}$ is planar and $\mathrm{Au}_{8}$ already presents a 3D structure, while the 2D/3D transition using DFT calculations occurs at $N=11$ (Ref. 12) because DFT methods underestimate long range interactions. Recently, new $\operatorname{CCSD}(\mathrm{T})$ calculation on $\mathrm{Au}_{10}$ (Ref. 28) agrees with the previous results, but they found that the basis set superposition error corrections favor the 2D structures again. Nevertheless, the experimental results, by the complexity of the setup, needs some guidance from the theoretical simulations, which need to be as accurate as possible.

Reactivity on metallic clusters is very sensitive and requires high accuracy for the potential energy surfaces (PESs). However, high level $a b$ initio calculations are restricted to some small examples such as $\mathrm{Au}_{N}+\mathrm{H}_{2}$ with $N$ $=2,3$, and $4,{ }^{25,29}$ using a $\operatorname{CCSD}(\mathrm{T})$ method. Even if this number of gold atoms is small, in some cases it can be enough to get a qualitative idea about the different reactive processes taking place. For example, in recent calculations 
on the reactivity of $\mathrm{H}_{2}$ on linear gold chains, ${ }^{25}$ it is found that the minimum energy paths (MEPs) obtained for $\mathrm{Au}_{N}$ with $N \geq 4$ are similar, while for $N<4$ the reactivity is rather low. Thus, it is interesting to study the evolution from one to four gold atoms with the maximum accuracy, in order to analyze the evolution of the main factors affecting the reactivity of gold. Furthermore, this gives access to usual detailed gas phase experimental techniques, such as crossed beams, which allow the direct determination of products and starting the reaction for different electronic states of the metal atoms. $^{30-33}$

In this work, we start such a study with a detailed description of the $\mathrm{Au}\left({ }^{2} S\right)+\mathrm{H}_{2}\left({ }^{1} \Sigma_{g}^{+}\right)$reactive collisions. Previous theoretical work ${ }^{34}$ on this system investigated the three low-lying electronic states of $\mathrm{AuH}_{2},{ }^{2} \Sigma_{g}^{+},{ }^{2} \Sigma_{u}^{+}$, and ${ }^{2} B_{2}$, and more recently the electronic structure, stability, and spectroscopy of dihydrides of group-IB metals, ${ }^{35}$ but until now there is no available PES to study the reaction dynamics. Experimentally the $\mathrm{AuH}_{2}$ molecule has been formed in solid hydrogen by reactions of excited gold atoms from laser ablation and irradiation after thermal evaporation. ${ }^{36}$ Here we develop a global PES capable of describing the reaction dynamics along the collision, which is studied by means of quantum and quasiclassical calculations to get the state resolved differential cross sections (DCSs). This reaction is endoergic in the ground electronic state by more than $1.5 \mathrm{eV}$, while on the ${ }^{2} D$ or ${ }^{2} P$ excited states it is exothermic. When the number of gold atoms grows, it is possible to form the double bridge $\mathrm{Au}-\mathrm{H}-\mathrm{Au}-\mathrm{H}-\mathrm{Au}$ chemisorption well and the reaction barrier may disappear, as described for linear gold chains. ${ }^{25}$ Such reaction mechanism is probably due to the lowering of the excited electronic states because of the $\mathrm{Au}-\mathrm{Au}$ interactions. For this reason, in this work, we shall pay special attention to the excited states and to the description of the intersections among them.

This paper is organized as follows. First, in Sec. II, the $a b$ initio methodology used is outlined, and the global PES and electronic state intersections are described. In Sec. III, the dynamical results obtained are described. Finally, Sec. IV is devoted to extract some conclusions.

\section{AB INITIO CALCULATIONS AND FIT}

It is well known that relativistic effects are important in compounds containing heavy elements. In order to include these relativistic effects, we used for $\mathrm{Au}$ the one-electron Gaussian-type basis sets ECP60MDF of the Stuttgart pseudopotential group. ${ }^{37}$ This basis set describes the 60 core electrons of the Au with a small-core fully relativistic effective core potential and the remaining 19 electrons [8 semicore $\left(5 s^{2} 5 p^{6}\right)$ and 11 valence $\left(5 d^{10} 6 s^{1}\right)$ electrons] with a $(12 s 12 p 9 d 3 f 2 g) /[6 s 6 p 4 d 3 f 2 g]$ Gaussian basis set. In this work we use the spdf space. For the $\mathrm{H}$ atom the correlationconsistent polarized valence triple zeta basis set of Dunning $^{38}$ augmented with diffuse functions (aug-cc-pVTZ) is used.

All $a b$ initio electronic structure calculations were carried out with the MOLPRO suite of programs. ${ }^{39}$ As a starting point, a full valence state-averaged complete active space
TABLE I. Comparison of the energy levels of neutral gold.

\begin{tabular}{lcccc}
\hline \hline & & \multicolumn{3}{c}{ Energy level $(\mathrm{eV})$} \\
\cline { 3 - 5 } Configuration & Term & Exp. $^{\text {a }}$ & ic-MRCI & ic-MRCI(+Q) \\
\hline $5 d^{10} 6 s$ & ${ }^{2} S$ & 0 & 0 & 0 \\
$5 d^{9} 6 s^{2}$ & ${ }^{2} D$ & 1.744 & 1.692 & 1.862 \\
$5 d^{10} 6 p$ & ${ }^{2} P$ & 4.947 & 4.855 & 4.981 \\
\hline
\end{tabular}

${ }^{\mathrm{a}}$ The experimental atomic results are $J$-averaged atomic energy levels from Ref. 46.

MCSCF calculation ${ }^{40,41}$ (SA-CASSCF) was performed. The molecular orbitals (MOs) correlating with $5 d, 6 s$, and $6 p$ atomic orbitals of the $\mathrm{Au}$ atom are used to determine the valence space, because this allows to describe the ground electronic state, ${ }^{2} S$, and excited states, ${ }^{2} D$ and ${ }^{2} P$, of the $\mathrm{Au}$ atom. The four first MO corresponding approximately to the eight semicore $\left(5 s^{2} 5 p^{6}\right)$ orbitals of the gold atom, have been optimized, but maintaining them doubly occupied. All calculations were performed in $C_{s}$ symmetry, to avoid the use of different active space in the CASSCF. The SA-CASSCF wave function is optimized with respect to the nine states ( $\operatorname{six}{ }^{1} A^{\prime}$ and three ${ }^{1} A^{\prime \prime}$ ) that asymptotically correlate with $\mathrm{Au}\left({ }^{2} S\right), \mathrm{Au}\left({ }^{2} D\right)$, and $\mathrm{Au}\left({ }^{2} P\right)+\mathrm{H}_{2}\left(\mathrm{X}^{1} \Sigma_{g}^{+}\right)$.

With the SA-CASSCF orbitals, internally contracted multireference configuration interaction (icMRCI) calculations $^{42,43}$ with single and double excitations have been done, using all the SA-CASSCF configurations as reference functions. Only four MOs are considered in the core in the icMRCI calculations (three $a^{\prime}$ and one $a^{\prime \prime}$ ). A total of four ${ }^{1} A^{\prime}$ and two ${ }^{1} A^{\prime \prime}$ states have been calculated, that asymptotically correlate with $\mathrm{Au}\left({ }^{2} S\right)$ and $\mathrm{Au}\left({ }^{2} D\right)+\mathrm{H}_{2}\left(\mathrm{X}^{1} \Sigma_{g}^{+}\right)$. These states correlate with three $A_{1}$, one $A_{2}$, one $B_{1}$, and one $B_{2}$ states in $C_{2 v}$. [For the analysis of the curve crossings presented below, also other excited electronic states have been considered, correlating with the $\mathrm{Au}\left({ }^{2} P\right)$ asymptote.) With this state selection the icMRCI calculations involve a number of contracted (uncontracted) configurations of the order of $2.8 \times 10^{6}\left(175 \times 10^{6}\right)$ for the ${ }^{2} A^{\prime}$ states and $2.3 \times 10^{6}$ $\left(174 \times 10^{6}\right)$ for the ${ }^{2} A^{\prime \prime}$ states. Finally, a multireference Davidson correction technique ${ }^{44}$ (icMRCI+Q) was applied to the final energies in order to approximately account for unliked cluster effects of higher excitations.

The accuracy of the $a b$ initio computations with the relativistic pseudopotential at the dissociation asymptotes can be assessed $^{45}$ by comparing the calculated spectroscopic data with the experimental values, for the gold atom and for the diatomic molecules, $\mathrm{AuH}$ and $\mathrm{H}_{2}$. Table I shows the icMRCI, icMRCI(+Q), and experimental ${ }^{46}$ atomic energies for the ${ }^{2} S$, ${ }^{2} D$, and ${ }^{2} P$ levels of Au, taking the ground electronic state ${ }^{2} S$ as reference. Both the icMRCI method and the Davidson correction $(+\mathrm{Q})$ give excellent agreement with the experimental $J$-averaged atomic energy levels. ${ }^{46}$ Table II shows the spectroscopic constants for the ground electronic states of the $\mathrm{AuH}\left(\mathrm{X}^{1} \Sigma^{+}\right)$and $\mathrm{H}_{2}\left(\mathrm{X}^{1} \Sigma_{g}^{+}\right)$diatomic molecules, calculated in the supermolecule approach with the other atom $(\mathrm{H}$ and $\mathrm{Au}$, respectively) at a distance of $10 \AA$. Our results agree with experimental $^{47}$ and calculated values. ${ }^{35}$ 
TABLE II. Comparison of the $\mathrm{AuH}\left(\mathrm{X}^{1} \Sigma^{+}\right)$and $\mathrm{H}_{2}\left(\mathrm{X}^{1} \Sigma_{g}^{+}\right)$spectroscopic constants.

\begin{tabular}{lcccc}
\hline \hline Diatom & Property & Exp. $^{\text {a }}$ & ic-MRCI & ic-MRCI(+Q) \\
\hline \multirow{2}{*}{ AuH } & $r_{e} / \AA$ & 1.524 & 1.526 & \\
& $\omega_{e} / \mathrm{cm}^{-1}$ & 2305 & 2239 & \\
& $D_{e} / \mathrm{eV}$ & 3.36 & 2.93 & 3.07 \\
$\mathrm{H}_{2}$ & $r_{e} / \AA$ & 0.7414 & 0.7426 & \\
& $\omega_{e} / \mathrm{cm}^{-1}$ & 4401 & 4410 & \\
& $D_{e} / \mathrm{eV}$ & 4.74 & 4.55 & 4.74 \\
\hline \hline
\end{tabular}

${ }^{a}$ Experimental results from Ref. 47.

\section{A. Global potential energy surface}

$A b$ initio calculations have been performed in a grid in Jacobi reactants coordinates, defined by the vector $\mathbf{r}$ between the two hydrogen atoms, and the vector $\mathbf{R}$ that points from the $\mathrm{H}_{2}$ center of mass to the $\mathrm{Au}$ atom. The grid of points is defined by the modulus $r$ and $R$ of the two Jacobi vectors, and the angle $\gamma$, defined by $\cos \gamma=\mathbf{r} \cdot \mathbf{R} / r R$,

$$
\begin{aligned}
& r=0.4-5.0 \AA(22 \text { values }), \\
& R=0.0-5.0 \AA(12 \text { values }), \\
& \gamma=0-\pi / 2(9 \text { values }),
\end{aligned}
$$

which gives a total of 2376 ab initio points. Finally, points with high energy were discarded, giving 1763 calculated points.

The $a b$ initio icMRCI+Q energies for the ground electronic state, ${ }^{2} A^{\prime}$, has been fitted using the GFIT3C procedure introduced in Refs. 48-50. The global PES is represented by a many-body expansion,

$$
V_{A B C}=\sum_{A} V_{A}^{(1)}+\sum_{A B} V_{A B}^{(2)}\left(r_{A B}\right)+V_{A B C}^{(3)}\left(r_{A B}, r_{A C}, r_{B C}\right),
$$

where $V_{A}^{(1)}$ represents the energy of the atoms $(A=\mathrm{Au}, \mathrm{H}, \mathrm{H})$ in the ground electronic state, $V_{A B}^{(2)}$ the diatomic terms $(A B=\mathrm{AuH}, \mathrm{AuH}, \mathrm{HH})$, and $V_{A B C}^{(3)}$ the triatomic one $(A B C=\mathrm{AuHH})$.

The diatomic terms are written as a sum of short- and long-range contributions. The short-range potential is defined as a shielded Coulomb potential, whereas the long-range term is a linear combination of modified Rydberg functions ${ }^{51}$ defined as

$$
\rho_{A B}\left(r_{A B}\right)=r_{A B} e^{-\beta_{A B}^{(2)} r_{A B}}, \quad A B=\mathrm{AuH}, \mathrm{AuH}, \mathrm{HH},
$$

with $\beta_{A B}^{(2)}>0$. The root-mean-square (rms) error of the fitted potentials from $a b$ initio values are 1.4 and $1.8 \mathrm{meV}$, for $\mathrm{AuH}\left(\mathrm{X}^{1} \Sigma^{+}\right)$and $\mathrm{H}_{2}\left(\mathrm{X}^{1} \Sigma_{g}^{+}\right)$, respectively.

The three-body term is expressed as an expansion,

$$
V_{A B C}^{(3)}\left(r_{A B}, r_{A C}, r_{B C}\right)=\sum_{i j k}^{K} d_{i j k} \rho_{A B}^{i} \rho_{A C}^{j} \rho_{B C}^{k},
$$

in the same type of modified Rydberg functions, but with exponents $\beta_{A B}^{(3)}>0$. For $A B B$ systems, the $\mathrm{AuH}_{2}$, there are only two nonlinear parameters, $\beta_{\mathrm{AuH}}^{(3)}$ and $\beta_{\mathrm{HH}}^{(3)}$, and additional constraints $^{48-50}$ in the linear parameters, $d_{i j k}$. The linear pa-
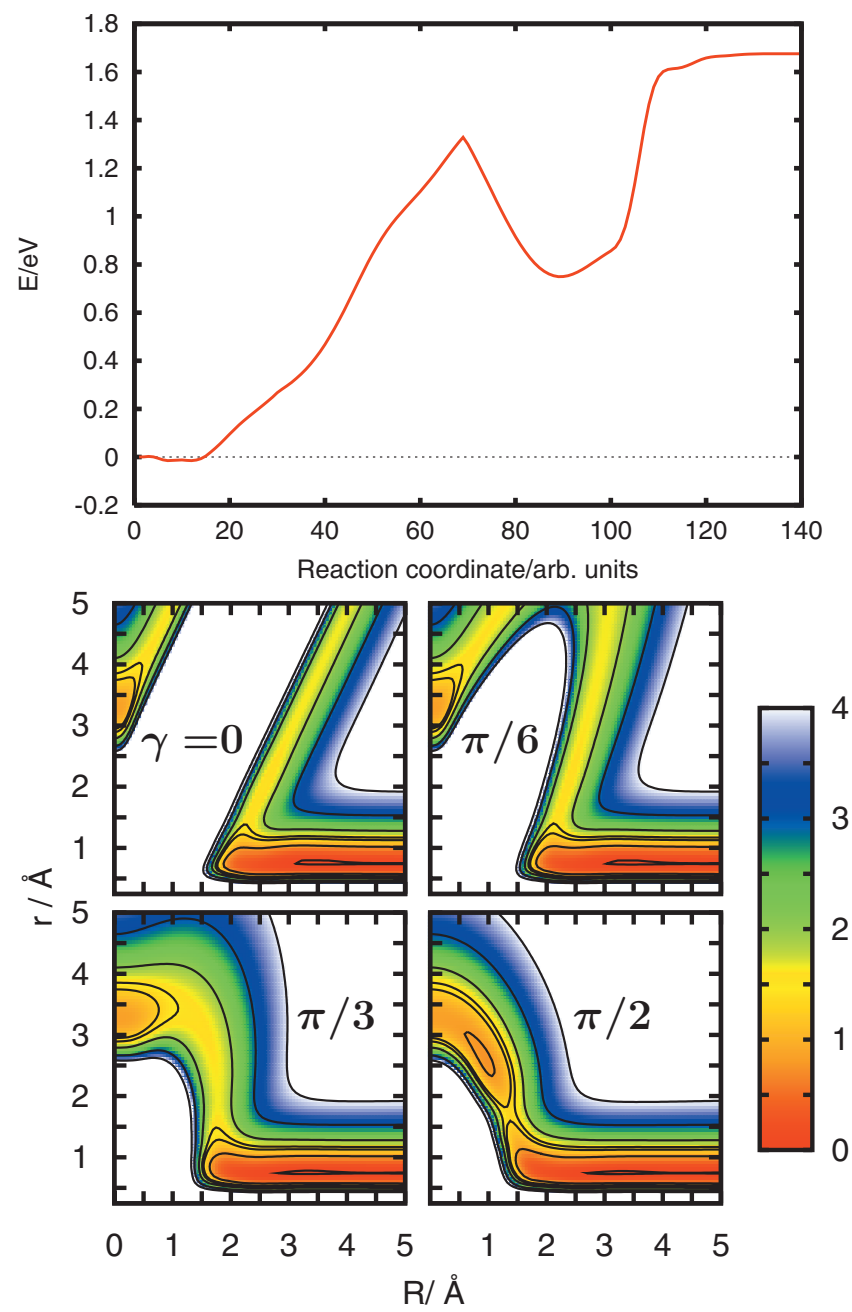

FIG. 1. Minimum energy path (top panel) in eV for the PES of the ${ }^{2} A^{\prime}$ ground state of $\mathrm{AuH}_{2}$. Four lower panels: contour plots of the PES as a function of the two Jacobi distances, $r$ and $R$ obtained in reactant Jacobi coordinates for the angles $\gamma=0, \pi / 6, \pi / 3$, and $\pi / 2$ (with $\cos \gamma=\mathbf{r} \cdot \mathbf{R} / r R$ ). The contours correspond to $0,0.8,1.3,1.5,2,3$, and $4 \mathrm{eV}$.

rameters $d_{i j k}(i+j+k \leq L)$ and the two nonlinear parameters, $\beta_{\mathrm{AuH}}^{(3)}$ and $\beta_{\mathrm{HH}}^{(3)}$, are determined by fitting the 1763 calculated $a b$ initio energies after subtraction of the one- and two-body contributions. The overall rms error of the fitted PES in the $1763 a b$ initio points is $38 \mathrm{meV}$.

\section{B. Topological characteristics}

The main topological characteristics of the fitted PES are shown in Fig. 1, where the minimum energy path from reactants $\mathrm{Au}\left({ }^{2} S\right)+\mathrm{H}_{2}\left(\mathrm{X}^{1} \Sigma_{g}^{+}\right)$to products $\mathrm{AuH}\left(\mathrm{X}^{1} \Sigma^{+}\right)+\mathrm{H}\left({ }^{2} S\right)$ is shown together with contour plots for several angles, in reactant Jacobi coordinates. For angles near collinear geometries there is a simpler 2D-MEP, in which energy monotonously increases when evolving from reactants to products. In nearly T-shaped geometries, the gold atom inserts in between the two hydrogen atoms, forming a well with an isosceles configuration, with an $\mathrm{Au}-\mathrm{H}_{2}$ angle of $\pi / 2$, in a $C_{2 v}$ geometry. This well is considerably above $(\approx 0.8 \mathrm{eV})$ the $\mathrm{Au}+\mathrm{H}_{2}$ reactants. These features indicate that there may be two different reaction mechanisms, one direct at nearly collinear geometries, and another indirect, in which an insertion 
TABLE III. Stationary points of the $\mathrm{AuH}_{2}$ fitted ground electronic state.

\begin{tabular}{lcccc}
\hline \hline Stationary point & $\begin{array}{c}R_{\mathrm{HH}} \\
(\AA)\end{array}$ & $\begin{array}{c}R_{\mathrm{AuH}} \\
(\AA)\end{array}$ & $\begin{array}{c}\theta \\
(\mathrm{deg})\end{array}$ & $\begin{array}{c}\text { Energy } \\
(\mathrm{eV})\end{array}$ \\
\hline Reactants: $\mathrm{Au}\left({ }^{2} S\right)+\mathrm{H}_{2}\left(\mathrm{X}^{1} \Sigma_{g}^{+}\right)$ & 0.7425 & $\ldots$ & $\ldots$ & 0.00 \\
Products: $\mathrm{AuH}\left({ }^{1} \Sigma^{+}\right)+\mathrm{H}\left({ }^{2} S\right)$ & $\ldots$ & 1.526 & $\ldots$ & 1.66 \\
Transition state & 1.704 & 1.585 & 57.52 & 1.34 \\
Minimum & 2.591 & 1.602 & 36.03 & 0.737 \\
\hline \hline
\end{tabular}

complex is formed prior to the formation of products. The geometries, in bond coordinates, and energies of the stationary points of the fitted ${ }^{2} A^{\prime}$ PES are presented in Table III. Our result for the height of the barrier, $1.34 \mathrm{eV}$, is lower that the previous ones, ${ }^{35,36}$ of 1.43 and $1.85 \mathrm{eV}$.

The barrier is a consequence of a conical intersection (CI) between the lowest ${ }^{2} A_{1}$ and ${ }^{2} B_{2}$ states in the $C_{2 v}$ point group, called CI-1 in Fig. 2 at $R \approx 1.3 \AA$, and its height is very sensitive to the relative position of the atomic levels of the gold atom (Table I). In order to better characterize this CI it should be taken into account that there are some more CIs between the excited electronic states. In Fig. 2 a second CI (called CI-2) is shown between two excited ${ }^{2} B_{2}$ states, occurring at $R \approx 1.9 \AA$, and correlating with the $\mathrm{Au}\left({ }^{2} P\right)$ and $\mathrm{Au}\left({ }^{2} D\right)$ electronic states. Gold in the ground ${ }^{2} S$ state is not reactive, while in ${ }^{2} D$ and ${ }^{2} P$ is reactive. Thus the mixing between those states of atomic gold occurring at these CIs makes the resulting ground adiabatic state correlate with $\mathrm{Au}\left(\Sigma^{+}\right)$products. Also, these CIs are the responsible for the appearance of the insertion well, leading to the indirect reaction mechanism discussed below. This well, which is higher in energy than the $\mathrm{Au}+\mathrm{H}_{2}$ asymptote, could be the analog to the much deeper chemisorption well appearing in larger gold clusters reacting with $\mathrm{H}_{2}$.

The two excited electronic states involved in CI-2 have the same irreducible representation ${ }^{2} B_{2}$ in the $C_{2 v}$ symmetry.

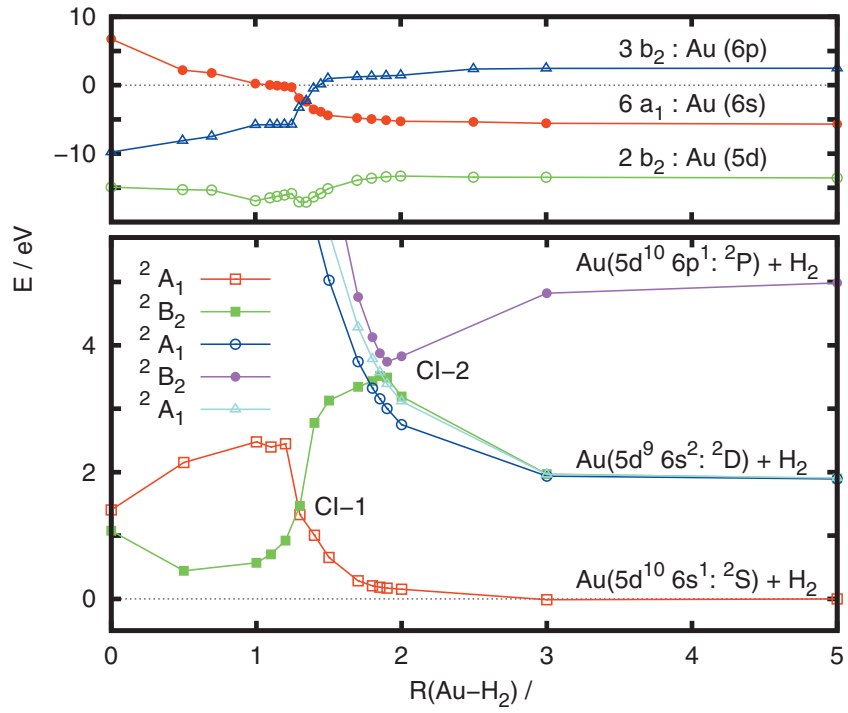

FIG. 2. Lower panel: icMRCI+Q calculated MEP for the insertion of $A u$ $+\mathrm{H}_{2}$ in $C_{2 v}$. Only the states of ${ }^{2} A_{1}$ and ${ }^{2} B_{2}$ symmetry are displayed for clarity. Top panel: energy of the $6 a_{1}, 2 b_{2}$, and $3 b_{2}$ orbitals obtained along the MEP. The contributions of the Au atomic orbitals at long distances are included.
TABLE IV. Electronic configurations and main coefficients for the expansion of the icMRCI wave function for the lowest electronic states of the $\mathrm{AuH}_{2}$ in the $C_{2 v}$ point group at the conical intersection between the $1{ }^{2} B_{2}$ and $2{ }^{2} B_{2}$ states.

\begin{tabular}{lccc}
\hline \hline Configurations ${ }^{\mathrm{a}}$ & $1{ }^{2} A_{1}$ & $1{ }^{2} B_{2}$ & $2{ }^{2} B_{2}$ \\
\hline \multicolumn{4}{c}{$R=5.00 \AA$} \\
$\cdots\left(5 a_{1}\right)^{2}\left(2 b_{2}\right)^{2}\left(6 a_{1}\right)^{1}$ & 0.989 & & \\
$\cdots\left(5 a_{1}\right)^{1}\left(2 b_{2}\right)^{2}\left(6 a_{1}\right)^{2}$ & 0.003 & & \\
$\cdots\left(5 a_{1}\right)^{2}\left(2 b_{2}\right)^{1}\left(6 a_{1}\right)^{2}$ & & 0.972 & 0.000 \\
$\cdots\left(5 a_{1}\right)^{2}\left(2 b_{2}\right)^{2}\left(3 b_{2}\right)^{1}$ & & 0.000 & 0.942 \\
& & & \\
$\cdots\left(5 a_{1}\right)^{2}\left(2 b_{2}\right)^{2}\left(6 a_{1}\right)^{1}$ & 0.953 & & \\
$\cdots\left(5 a_{1}\right)^{1}\left(2 b_{2}\right)^{2}\left(6 a_{1}\right)^{2}$ & 0.211 & & \\
$\cdots\left(5 a_{1}\right)^{2}\left(2 b_{2}\right)^{1}\left(6 a_{1}\right)^{2}$ & & -0.701 & 0.668 \\
$\cdots\left(5 a_{1}\right)^{2}\left(2 b_{2}\right)^{2}\left(3 b_{2}\right)^{1}$ & & 0.661 & 0.693 \\
& & & \\
$\cdots\left(5 a_{1}\right)^{2}\left(2 b_{2}\right)^{2}\left(6 a_{1}\right)^{1}$ & 0.962 & & \\
$\cdots\left(5 a_{1}\right)^{1}\left(2 b_{2}\right)^{2}\left(6 a_{1}\right)^{2}$ & 0.143 & & \\
$\cdots\left(5 a_{1}\right)^{2}\left(2 b_{2}\right)^{1}\left(6 a_{1}\right)^{2}$ & & -0.103 & 0.917 \\
$\cdots\left(5 a_{1}\right)^{2}\left(2 b_{2}\right)^{2}\left(3 b_{2}\right)^{1}$ & & 0.963 & 0.104 \\
& & & \\
$\cdots\left(5 a_{1}\right)^{1}\left(2 b_{2}\right)^{2}\left(3 b_{2}\right)^{2}$ & $R=0.50 \AA$ & & \\
$\cdots\left(5 a_{1}\right)^{1}\left(2 b_{2}\right)^{2}\left(6 a_{1}\right)^{2}$ & 0.848 & & \\
$\cdots\left(5 a_{1}\right)^{2}\left(2 b_{2}\right)^{1}\left(3 b_{2}\right)^{2}$ & 0.000 & & \\
$\cdots\left(5 a_{1}\right)^{2}\left(2 b_{2}\right)^{2}\left(3 b_{2}\right)^{1}$ & & & \\
\hline & & & \\
\hline
\end{tabular}

${ }^{\mathrm{a}}$ The $\cdots \quad$ represents MOs doubly occupied in all the states: $\left(1 a_{1}\right)^{2}\left(2 a_{1}\right)^{2}\left(1 b_{1}\right)^{2}\left(1 b_{2}\right)^{2}\left(3 a_{1}\right)^{2}\left(4 a_{1}\right)^{2}\left(2 b_{1}\right)^{2}\left(1 a_{2}\right)^{2}$.

As explained by Ruedenberg and co-workers, ${ }^{52}$ the intersection space (ICS) in this case is a line in the full $C_{s}$ space, while in the $2 \mathrm{D} C_{2 v}$ coordinate subspace is a point. In CI-1, however, the two states involved belong to different irreducible representations in the $C_{2 v}$ symmetry, ${ }^{2} A_{1}$ and ${ }^{2} B_{2}$, respectively. In this case, the ICS seam ${ }^{52}$ is a line in $C_{s}$ and in $C_{2 v}$ symmetry spaces. In order to check that there is a real CI between these two ${ }^{2} B_{2}$ states, the point of degeneracy has been searched, reaching a minimum energy difference of $10^{-4}$ a.u. at a $C_{2 v}$ configuration with $r=1.807 \AA$ and $R=3.833 \AA$. Dealing with states of the same symmetry, numerical calculations present difficulties to asses that two different states have the same energy with no doubts. That this is a CI or an avoided crossing is not essential for the discussion presented below, and we will denote it as CI-2. The interest is in the change of the electronic character occurring in this region, as will be discussed below. A deeper analysis of this crossing should be performed in order to study the reaction dynamics from excited electronic states.

In order to understand the reaction mechanism it is instructive to follow the electronic character of the electronic states involved in the different crossings and conical intersections along the reaction energy path. For this purpose, we first analyze the coefficients of the different electronic configurations of the $1{ }^{2} A_{1}, 1{ }^{2} B_{2}$, and $2{ }^{2} B_{2}$ states, and the main ones are listed in Table IV. In all the states and all along the MEP, the main configurations involve different occupation numbers in three orbitals, $6 a_{1}, 2 b_{2}$, and $3 b_{2}$, whose energies are shown in the top panel of Fig. 2. The $2 b_{2}$ orbital remains nearly constant in energy along the MEP, while the energies 


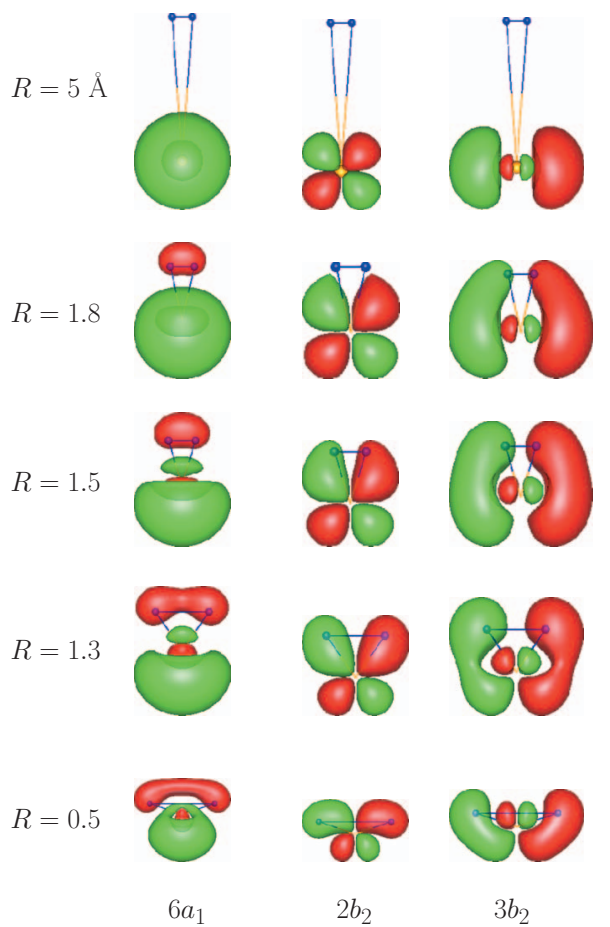

FIG. 3. Amplitudes of the $6 a_{1}, 2 b_{2}$, and $3 b_{2}$ for different $R$ values along the MEP of Fig. 2.

of $6 a_{1}$ and $3 b_{2}$ orbitals cross, thus explaining the change of configuration taking place at the CI-1. Note that the two states in CI-1, belonging to different irreducible representations in the $C_{2 v}$ symmetry group considered in the figure, become of the same symmetry in $C_{s}$ symmetry, so that the ground electronic state energy corresponds to the lower energy of these two states. In CI-2, the orbitals do not change, but their occupation numbers do, thus explaining the avoiding crossing.

To further analyze the electronic character of the states, the amplitude associated with the three orbitals are shown in Fig. 3. Clearly, at the asymptote, $R=5 \AA$, the $6 a_{1}, 2 b_{2}$, and $3 b_{2}$ orbitals correspond to the $6 s, 5 d$, and $6 p$ atomic orbitals of the gold atom. As the distance $R$ decreases, the orbitals change to form bonds with hydrogen atoms, but their $\mathrm{Au}$ atomic character remains essentially the same.

Thus, the insertion well in the ground adiabatic state corresponds to a $\cdots\left(5 a_{1}\right)^{2}\left(2 b_{2}\right)^{2}\left(3 b_{2}\right)^{1}$ electronic configuration. The doubly occupied $2 b_{2}$ orbital corresponds to a $\mathrm{Au}(5 d)$ orbital, bonding with respect to the $\mathrm{Au}-\mathrm{H}$ bonds and antibonding with respect to the $\mathrm{H}-\mathrm{H}$ bond. The singly occupied $3 b_{2}$ orbital corresponds to a $\mathrm{Au}(6 p)$ orbital, with similar bonding/antibonding character. Even when this orbital is singly occupied, it plays a crucial role in the stabilization of the well, because its orbital energy decreases considerably at this geometry. There is a connection between this insertion well and the much deeper chemisorption well appearing in larger gold clusters: the so called $\mathrm{Au}-\mathrm{H}-\mathrm{Au}-\mathrm{H}-\mathrm{Au}$ double-bridge bond $^{25}$ is further stabilized by the formation of other bonds between the hydrogen atoms and the neighbor gold atoms. It is therefore interesting to study the evolution of this well in $\mathrm{Au}_{N}+\mathrm{H}_{2}$ systems, as $N$ increases.

Finally, the energy of AuH products is $\approx 1.66 \mathrm{eV}$, and is
TABLE V. Parameters used in the WP calculations in reactant Jacobi coordinates for the $\mathrm{Au}+\mathrm{H}_{2}$ reaction.

\begin{tabular}{lc}
\hline \hline$r_{\min } / \AA$ & 0.01 \\
$r_{\max } / \AA$ & 15 \\
$N_{r}$ & 256 \\
$r_{I} / \AA$ & 11 \\
$A_{r} / \AA^{-2}$ & 0.05 \\
$R_{\min } / \AA$ & 0.01 \\
$R_{\max } / \AA$ & 15 \\
$N_{R}$ & 640 \\
$R_{I} / \AA$ & 11 \\
$A_{R} / \AA \AA^{-2}$ & 0.05 \\
$N_{\gamma}$ & 104 in $[0, \pi] / 2$ \\
$R_{0} / \AA$ & 10 \\
$E_{0} / \mathrm{eV}$ & 1.75 \\
$\Delta E / \mathrm{eV}$ & 0.25 \\
$R_{\infty}^{\prime}$ & 10 \\
$V_{\text {cut }} / \mathrm{eV}$ & 5 \\
$E_{\text {cut }}^{\ell} / \mathrm{eV}$ & 5 \\
\hline \hline
\end{tabular}

directly correlated with the $1^{2} A_{1}$ state at $R=0$. Thus there is another curve crossing in the exit channel, between the $1^{2} A_{1}$ and $1^{2} B_{2}$ states. The bonding orbitals of the $\operatorname{AuH}\left({ }^{1} \Sigma\right)$ state essentially corresponds to a $\mathrm{Au}(5 d)$ orbital at the equilibrium distance, while at long $\mathrm{Au}-\mathrm{H}$ distances it is mainly formed with the $\mathrm{Au}(6 s)$ orbital.

\section{REACTION DYNAMICS}

\section{A. Quantum wave packet propagation}

The quantum wave packet (WP) method used here has been described in detail elsewhere ${ }^{53-55}$ and will be briefly outlined here for clarity. Basically, the WP is represented in a grid in reactant Jacobi coordinates, described above, and it is propagated in time using a dumped Chebyshev propagator. ${ }^{56-61}$ The total reaction probability is obtained analyzing the flux on the Chebyshev components at a long $r$ value. ${ }^{61}$ The state-to-state reaction probabilities are obtained by transforming the WP at each iteration from reactant to product Jacobi coordinates, where it is projected on the asymptotic states of the products, following an efficient method recently proposed. ${ }^{62}$ The calculations are performed using the code MAD-WAVE3 described in Ref. 63. The parameters of the calculations performed here for zero total angular momentum, $J=0$, for the $\mathrm{Au}+\mathrm{H}_{2}(v=0, \quad j=0)$ $\rightarrow \mathrm{AuH}\left(v^{\prime}, j^{\prime}\right)+\mathrm{H}$ reaction are summarized in Table $\mathrm{V}$.

\section{B. The quasiclassical trajectory method}

In the quasiclassical trajectory (QCT) method, the generalized coordinates and momenta are propagated classically and quantization is introduced in the calculation by assigning vibration and rotation quantum numbers $v$ and $j$ to the reactant and product diatomic molecule. To get realistic results, the QCT calculations employed a standard Monte Carlo sampling of the initial conditions following the method introduced by Karplus et al. ${ }^{64}$ All the calculations were done with the code implemented by Halvick and Rayez. ${ }^{65}$ As for the quantum calculation, initially $\mathrm{H}_{2}$ is set in its ground rovibrational state for collision energies sampled between 1.5 and 3 
$\mathrm{eV}$. The step adaptive Adams method (average step $0.05 \mathrm{fs}$ ) is used to integrate the set of Hamilton equations, and the conservation of both total energy and angular momentum were carefully checked. At each step, relative precision of $10^{-8}$ for the distances and the momenta have been required, yielding to a conservation of total energy and total angular momentum with average errors of $10^{-4} \mathrm{eV}$ and $10^{-5} \hbar$, respectively. The high number of trajectories per batch, $N$ $=100000$ for the reaction at $J=0$ (impact parameter $b=0$ ) and $N=500000$ when including all angular momenta and/or impact parameter give a maximal statistical Monte Carlo error on reaction probability of $0.3 \%$ and $0.07 \%$, respectively. In order to have negligible long range effects, the initial distance between $\mathrm{Au}$ and $\mathrm{H}_{2}$ is set to $30 \AA$ and trajectories are stopped when the distance between products is larger than 10 $\AA$ and the recoil energy difference between steps is less than $10^{-7} \mathrm{eV}$. As the product channel is endoergic, the quantum numbers of the product molecule $v^{\prime}$ and $j^{\prime}$ have been assigned using Gaussian binning method ${ }^{66-69}$ with a half-width value set to 0.05 .

Once all trajectories are calculated, the $\mathrm{Au}+\mathrm{H}_{2}(v=0, \quad j=0) \rightarrow \mathrm{AuH}\left(v^{\prime}, j^{\prime}\right)+\mathrm{H} \quad$ reaction integral cross section for a given collision energy $E_{\text {col }}$ can then be written as

$$
\sigma_{v j \rightarrow v^{\prime} j^{\prime}}=\pi b_{\max }^{2} \frac{N_{v^{\prime} j^{\prime}}}{N_{t}},
$$

where $b_{\max }$ is the maximum value of the impact parameter leading to reaction, $N_{t}$ is the total number of trajectories with initial impact parameter lower than $b_{\max }$, and $N_{v^{\prime} j^{\prime}}$ is the number of trajectories leading to products in the final state $\left(v^{\prime}, j^{\prime}\right)$. For each reactive trajectory, the scattering angle $\theta_{c m}$ in the center of mass coordinate system is determined from the scalar product between the initial and final relative velocities vectors,

$$
\theta_{c m}=\pi-\arccos \left(\frac{\mathbf{V}_{\mathbf{r}} \cdot \mathbf{V}_{\mathbf{r}}^{\prime}}{V_{r} V_{r}^{\prime}}\right)
$$

and the DCS for a given scattering angle is calculated as

$$
\frac{d \sigma_{v j \rightarrow v^{\prime} j^{\prime}}}{d \Omega}=\frac{\sigma_{v j \rightarrow v^{\prime} j^{\prime}} P\left(\theta_{c m}\right)}{2 \pi \sin \left(\theta_{c m}\right)},
$$

where $P\left(\theta_{c m}\right)$ is the probability for the products in state $\left(v^{\prime}, j^{\prime}\right)$ to be scattered at the angle $\theta_{c m}$.

\section{Results and discussion}

\section{1. $\mathrm{J}=0$}

For $\mathbf{J}=0$, both quantum and QCT total reaction and state-to-state probabilities were calculated for collision energies going from 1 to $3 \mathrm{eV}$. In the top panel of Fig. 4, the total and vibrational resolved reaction probabilities obtained with the two methods are compared. The agreement is good in average, and they all show a similar threshold. Using the QCT method, the quantization of the initial state of the reactants is considered, but some attention should be paid with products. Since the zero-point energy (ZPE) of $\mathrm{AuH}$ is $\approx 0.14 \mathrm{eV}$, when using standard histogram binning the reac-
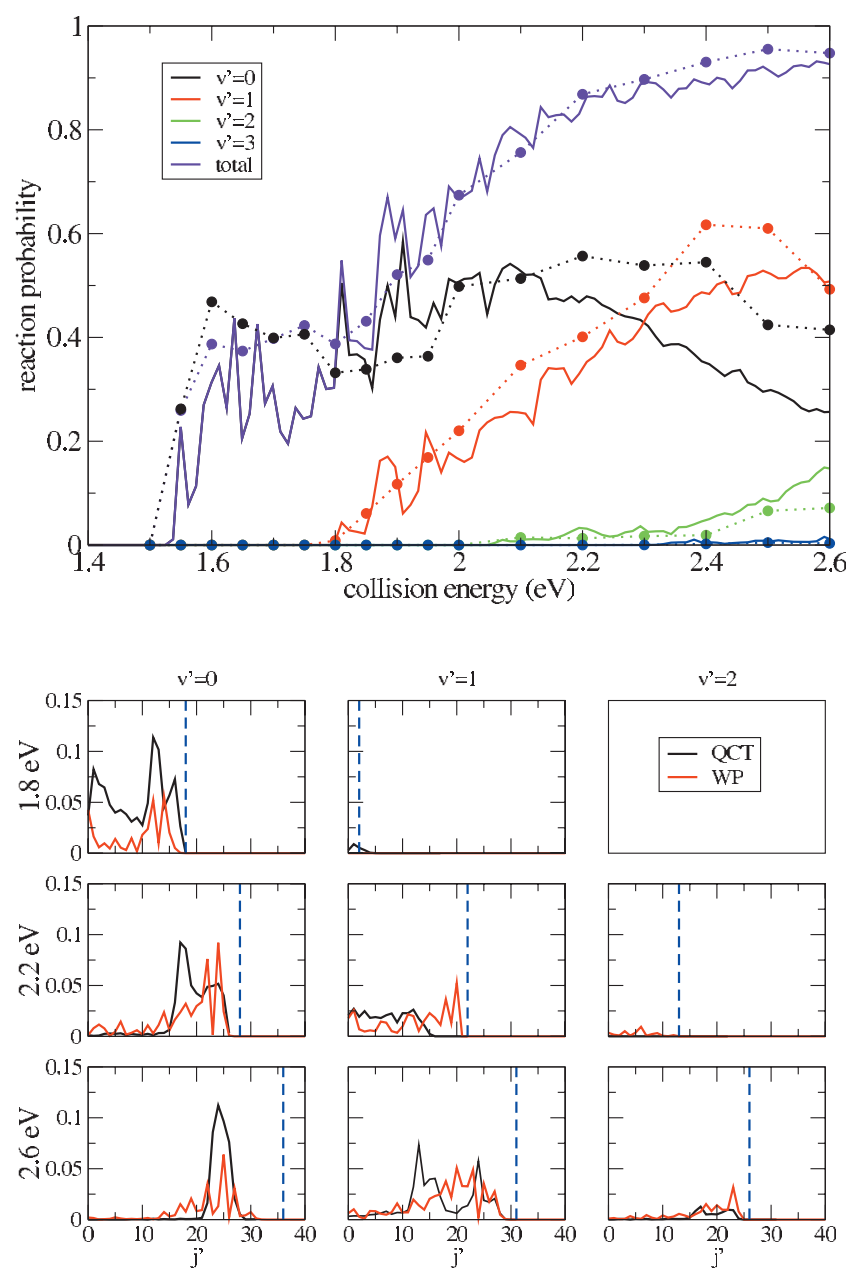

FIG. 4. Comparison between WP and QCT reaction probabilities obtained at total angular momentum $J=0$. Continue and dashed lines show WP and QCT results, respectively. Top panel: total and $\mathrm{AuH}\left(v^{\prime}\right)$ vibrational reaction probabilities as a function of collision energy. Bottom panel: rotational probabilities obtained using WP and QCT calculations for some selected energies and final vibrational state $v^{\prime}$.

tion threshold would be lowered by this amount since the ZPE of products is not taken into account. For exoergic reactions, however, since the final state of the products is rather high in energy, this effect is not important as recently reported for the $\mathrm{C}+\mathrm{OH} \rightarrow \mathrm{CO}+\mathrm{H}$ reaction. ${ }^{70-72}$ To account for the ZPE in this endothermic reaction, the Gaussian binning method ${ }^{6-69}$ is used, yielding an excellent agreement between QCT and WP calculations, with a threshold of 1.55 $\mathrm{eV}$. The barrier height along the MEP $(1.34 \mathrm{eV})$ is lower than the $\mathrm{AuH}+\mathrm{H}$ threshold $(1.66 \mathrm{eV})$, without including ZPEs. AuH ZPE $(0.138 \mathrm{eV})$ is lower than the one of $\mathrm{H}_{2}(0.271 \mathrm{eV})$, and adding the difference between the two ZPEs to the potential threshold energy gives exactly the energy threshold obtained in the WP and QCT calculations.

After the threshold, WP and QCT reaction probabilities increase (see the top panel of Fig. 4) and show an excellent agreement except that QCT results seems to overestimate slightly the probability of low $v^{\prime}$ for high energies. QCT reaction probabilities increase nearly monotonically, while the WP results show many oscillations typically associated with resonances, especially at low collision energies. The only well in the PES corresponds to the insertion $\mathrm{H}-\mathrm{Au}-\mathrm{H}$ 

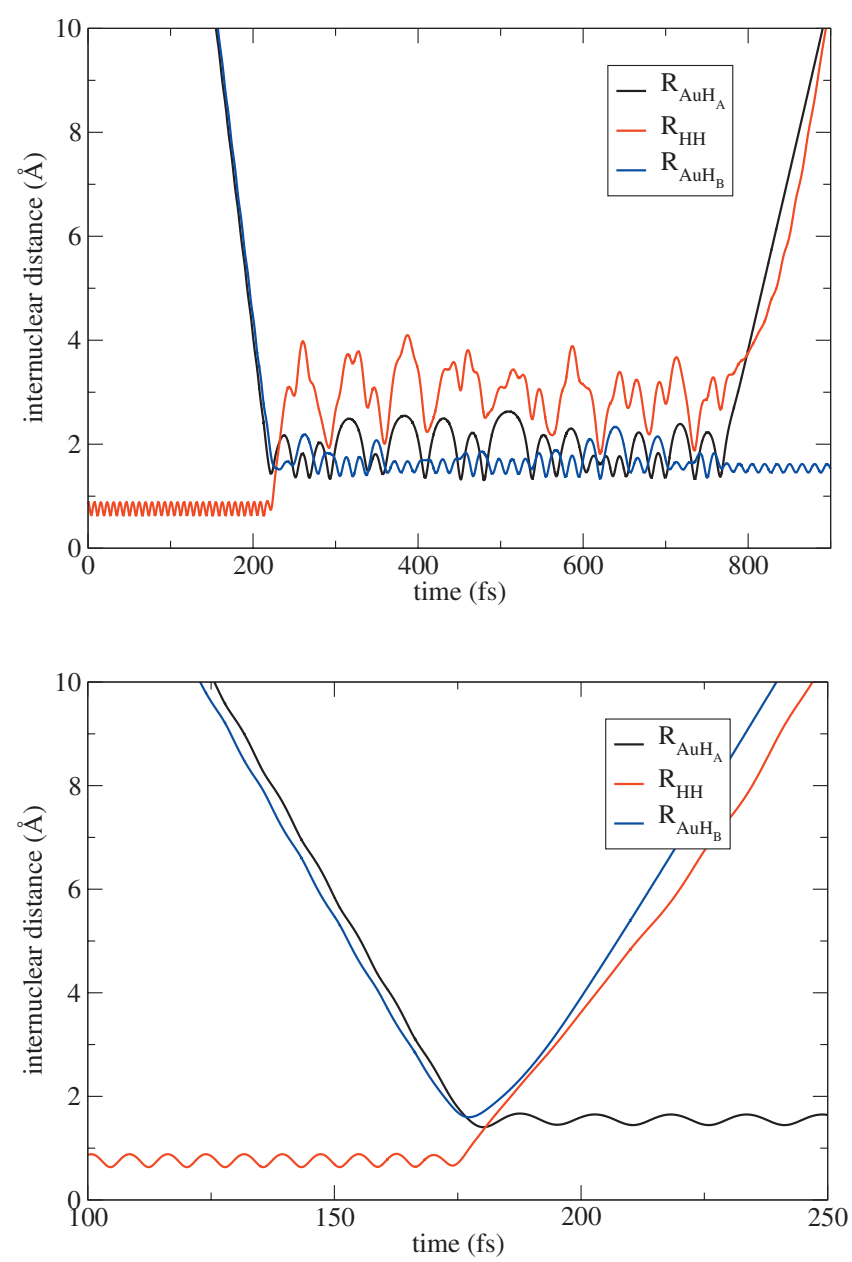

FIG. 5. Evolution of internuclear distances with time for a collision energy of $1.8 \mathrm{eV}$ (top panel) and $3 \mathrm{eV}$ (bottom panel).

complex, which is seen in Fig. 1 for $\gamma=\pi / 2$ and $\pi / 3$. For other Jacobi angles, near collinear geometry, there is a direct reaction path from reactants to products, in which the insertion well is not explored. Thus, for moderately high energies, the WP can move directly toward products, without "feeling" the presence of the well. At low energies, however, a portion of the WP can be "driven" by the potential to the insertion well, giving rise to resonances. Since the well is higher in energy than the reactant asymptote the resonances should have relatively short lifetimes, becoming broader with increasing energy. This, together with the natural increase of the density of states, makes the reaction probability become smoother as energy increases. In any case, the direct and insertion mechanisms can coexist.

Classically a similar process occurs. In fact, an analysis of the average lifetime of the complex (when the sum of internuclear distances is shorter than $6 \AA$ ) show that at energies near the threshold, at $1.7 \mathrm{eV}$, is of the order of $100 \mathrm{fs}$, while for $2.8 \mathrm{eV}$ the average lifetime is lower than $15 \mathrm{fs}$. To illustrate this, a long trajectory for $E=1.8 \mathrm{eV}$ and a short one for $3 \mathrm{eV}$ are shown in Fig. 5.

The final rotational distribution of products, in the bottom panel of Fig. 4, obtained with the QCT and WP methods also show a reasonably good agreement for most of the cases, worse than before simply because the quantities are now less averaged. In general, the $\mathrm{AuH}$ products are quite excited rotationally, the distribution being nonzero up for the maximum rotational quantum number $j^{\prime}$ available (marked with a dashed vertical line in the figure). Following an impulsive direct mechanism, the heavy Au drags one hydrogen atom in the collision, producing a high rotational excitation in this light atom, which orbits around the heavy Au atom very fast. If the reaction follows an insertion mechanism, on the other hand, the complex lives long time producing an important energy redistribution. In this case, a statistical distribution of fragments is expected, in which high $j^{\prime}$ are more probable because of the $2 j^{\prime}+1$ degeneracy.

It is also interesting to note the even/odd alternation of the quantum rotational population for higher $j$. Several convergence tests were done to avoid numerical problems, and the alternation structures were always obtained, even for the huge WP calculation done for $J=0$. A similar situation was obtained for the first rotational states of LiF products formed in the Li+HF collisions, ${ }^{73}$ and was explained by the symmetric angular potential at the transition state, in product Jacobi coordinates, which decouples even and odd rotational states. Here, the situation is different. The even/odd rotational symmetry exists in the entrance channel, because the system is symmetric under the exchange of hydrogen atoms, but not in the exit channel. Several models were tried with no success, and the only explanation is an interference between two different pathways, but some more work on this is required to confirm it.

Finally, the overall agreement between WP and QCT results allows us to assess that in this reaction there are no major quantum effects, and the dynamics can be reasonably well described using a QCT method. For this reason, in the following, the cross section for this reaction based only on QCT calculations will be presented and discussed, whose interest is in that these magnitudes are directly comparable with experimental measurements.

\section{2. $\boldsymbol{J} \neq 0$}

The total integral cross section, calculated with the QCT method, is shown in the top panel of Fig. 6 as a function of the collision energy, and presents a threshold of $\approx 1.55 \mathrm{eV}$, similar to that obtained for $J=0$. As energy increases, new vibrational channels $v^{\prime}$ open, but always the partial cross section toward $v^{\prime}=0$ is dominant, showing that the $T \rightarrow V$ energy transfer is not very efficient. The rotationally resolved cross sections, presented in the lower panels of Fig. 6, show a much higher and broad excitation. That the energy transfer goes essentially to rotation and no vibration of $\mathrm{AuH}$ products is clearly seen for $2.6 \mathrm{eV}$, in the lower panels Fig. 6, for which the partial cross section of the $\left(v^{\prime}=0, j^{\prime}=25\right)$ state is much larger than the one of $\left(v^{\prime}=1, \quad j^{\prime}=18\right)$ and $\left(v^{\prime}=2, \quad j^{\prime}=5,6\right)$ states which have similar energy. In fact, for $v^{\prime}=0$, the maximum of the distribution is near the closing or maximum energy available, and for $E=2.6 \mathrm{eV}$ the rotational distribution shows a nearly linear slope, which is close to a statistical distribution of levels with the typical $\left(2 j^{\prime}\right.$ +1 ) dependence.

This could be explained by the large mismatch between masses and volumes of $\mathrm{Au}$ and $\mathrm{H}_{2}$, respectively. $\mathrm{Au}$ "hits" 

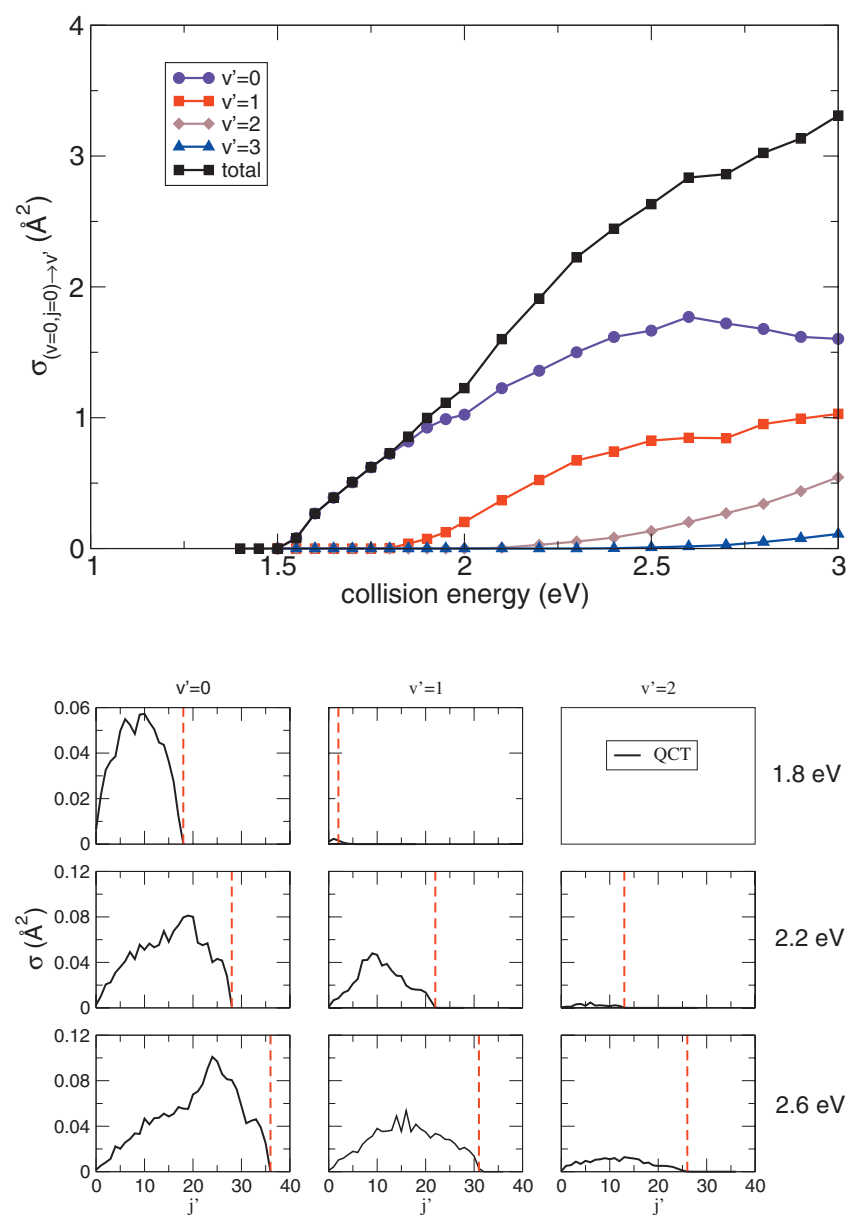

FIG. 6. $\mathrm{Au}+\mathrm{H}_{2}(v=0, j=0) \rightarrow \mathrm{AuH}\left(v^{\prime}, j^{\prime}\right)+\mathrm{H}$ reactive cross sections obtained with the QCT method. Top panel: total and vibrationally resolved reaction cross section as a function of collision energy. Bottom panel: rotationally resolved cross sections for some selected energies and $v^{\prime}$ states.

the ellipsoid $\mathrm{H}_{2}$ molecule pushing it as a whole and producing a high rotational excitation. If the reaction takes place, the Au atom "drags" one of the rotating $\mathrm{H}$ atoms, which continue rotating around the much heavier gold atom. The $\mathrm{AuH}$ vibration is not excited because the $\mathrm{Au}$ atom pushes $\mathrm{H}$ at the same speed it is traveling, forming a slowly vibrating AuH product, as can be seen in Fig. 5.

The maximum of the rotational distributions in Fig. 6 is similar to those obtained for $J=0$, in Fig. 4. The main change when increasing $J$ is therefore the broadening of the distribution. This could be explained by the value of the skewing angle $\beta$ [with $\cos ^{2} \beta=m_{A} m_{C} /\left(m_{B}+m_{C}\right)\left(m_{A}+m_{B}\right)$ for a $A+B C \rightarrow A b+C$ reaction], which in this case takes an intermediate value of $\approx 45^{\circ}$. Using the coordinate transformation from reactant to product Jacobi coordinates, product angular momentum can be expressed as ${ }^{74,75}$

$$
\mathbf{j}^{\prime}=\ell \sin ^{2} \beta+\mathbf{j} \cos ^{2} \beta+\mathbf{x} \cos ^{2} \beta,
$$

where $\ell$ is the end-over-end orbital angular momentum in reactant Jacobi coordinates, and $\mathbf{x}=m_{B}(r \dot{R}+R \dot{r})$ arises from the shift of the center-of-mass of the diatomic molecule in the coordinate transformation. ${ }^{74}$ In this case, $\beta$ has an intermediate value and the three terms contribute significantly. Thus, for $J=0$ and $j=0, \ell$ must also be zero, and therefore

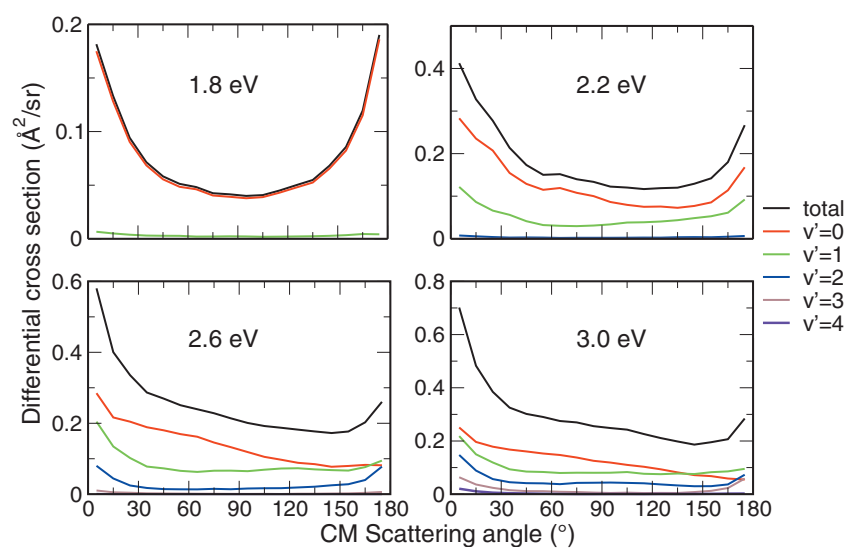

FIG. 7. Total and vibrationally resolved DCSs of the reaction $\mathrm{Au}+\mathrm{H}_{2}(v=0, j=0) \rightarrow \mathrm{AuH}\left(v^{\prime}\right)+\mathrm{H}$ for selected collision energies.

the excited rotational distribution should be essentially attributed to the $\mathbf{x}$ term due to the change of coordinates in this $\mathrm{H}+\mathrm{LL} \rightarrow \mathrm{HL}+\mathrm{L}$ reaction. When increasing $J, \ell$ also increases, and therefore the first term in the above expression also contributes, producing a broadening of the rotational distribution.

The behavior of the DCS as a function of energy presented in Fig. 7 indicates that the reaction mechanism changes with energy. At lower energies, the DCS is isotropic, with a nearly equivalent value for backward and forward scattering. As energy increases, the DCS gets more and more polarized toward forward scattering. In addition, the average lifetime of the complex decreases fast with increasing energy. All this indicate that at low collisional energy the process is indirect, proceeding through long-lived insertion resonances, while at high energies the mechanism is more likely a direct stripping reaction. This was illustrated in Fig. 5 by two typical trajectories, one leading to a long life complex for a collision energy of $1.8 \mathrm{eV}$ and to a short one for a collision energy of $3 \mathrm{eV}$.

What is surprising is that $\mathrm{AuH}$ can be produced in the backward direction, since its mass is of the order of 200 times that of hydrogen. The explanation is that, first, about $1.35-1.55 \mathrm{eV}$ of the relative translational energy of the system has to be converted into internal potential energy to allow the system to react and form AuH products. Second, if the insertion complex is formed, it lives a long time enough to produce some energy transfer, so that finally Au products can be formed backward. It seems, however, that there is always some backward scattering even at rather high energy, arising for the higher $v^{\prime}$ produced.

The efficiency of the transference of energy toward vibration, rotation, and translation of products is shown in the top panel of Fig. 8. Clearly, most of the energy goes into translation. However, this quantity is not as high as one would expect due to the large mass mismatch between $\mathrm{Au}$ and $\mathrm{H}_{2}$. At collision energies close to the threshold, the vibrational energy is slightly higher than the rotational one. This is not surprising since a classical energy has been considered, which includes the vibrational $\mathrm{ZPE}$ of $\mathrm{AuH}$. At a collision energy of $\approx 1.8$, however, the rotational energy fraction is larger and continues growing there after until 

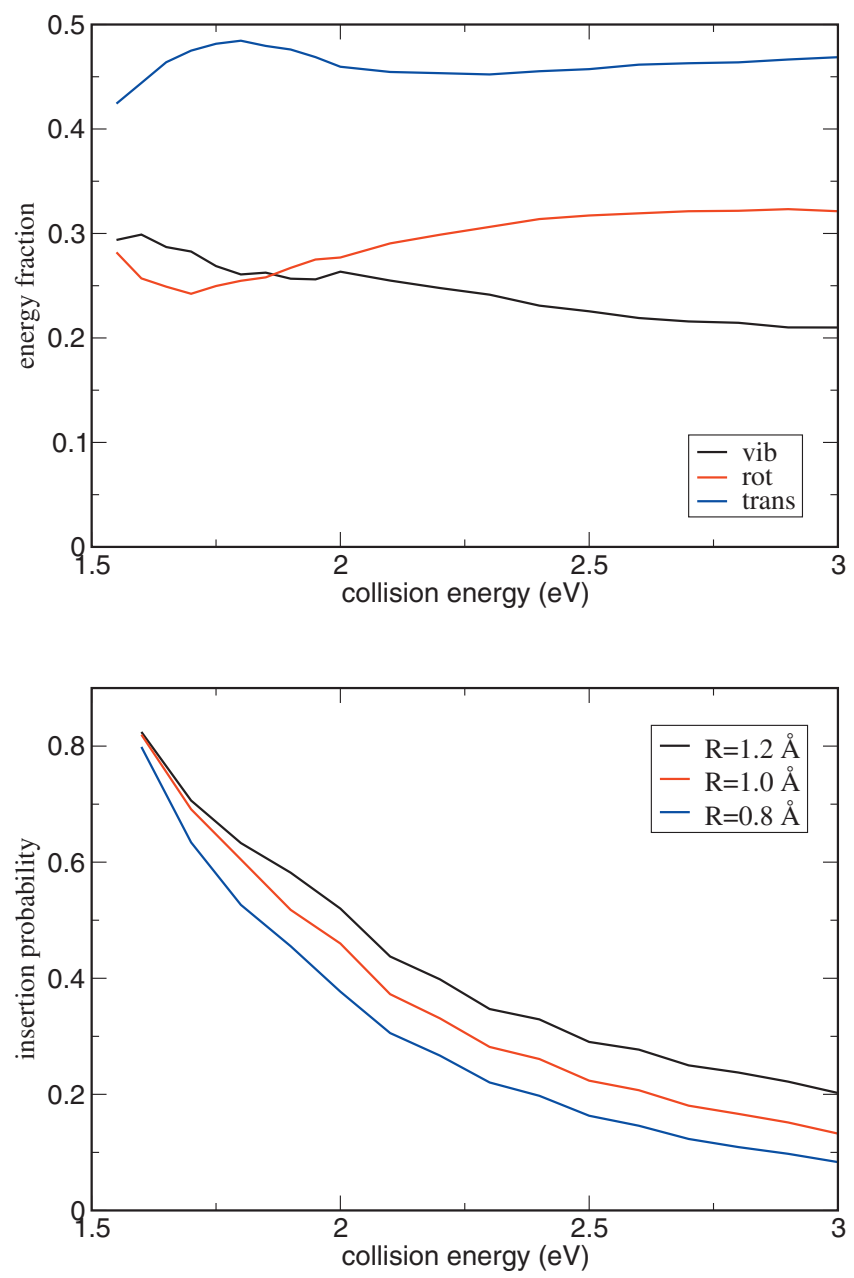

FIG. 8. Top panel: fraction of the available energy of products in vibration, rotation, or translation. Bottom panel: probability of reaching a given $R$ value, interpreted as the insertion probability, as a function of collision energy.

reaching a plateau at $2.6 \mathrm{eV}$ of more than $\frac{1}{3}$ of the available energy. This fraction is considerable and is explained by an impulsive mechanism, in which $\mathrm{Au}$ collides with the $\mathrm{H}_{2}$ ellipsoid at a bent geometry, producing a high rotational excitation of the $\mathrm{H}$ atoms which is maintained in the AuH products. Such high rotational excitation can also be obtained in an insertion mechanism, as discussed above.

The vibrational energy fraction, however, is always rather important, never less than $20 \%$. This suggests that there is always an important contribution from the insertion mechanism into the reaction dynamics, which originates high $v^{\prime}$, giving rise also to the backward peak in the DCS.

In order to quantify the occurrence of these two mechanisms an analysis of the trajectories has been done. To reach the insertion well, short $R$ values (shorter than $\approx 1 \AA$ ), are needed, as can be seen in Fig. 1. In the bottom panel of Fig. 8 , the probability of arriving to $R$ distances of $0.8,1$, and 1.2 $\AA$ are shown as a function of collision energy, calculated as the portion of trajectories reaching those $R$ values along the integration. They all show a similar decreasing behavior, illustrating the transition from insertionlike mechanism at low collision energies to a direct mechanism at higher energies, as discussed above.

\section{CONCLUSIONS}

The PES of the ground state of the $\mathrm{AuH}_{2}$ system has been calculated at the MRCI level and fit to generate an analytical global PES to study the $\mathrm{Au}\left({ }^{2} S\right)+\mathrm{H}_{2}$ reaction. This reaction is endoergic, by $1.55 \mathrm{eV}$, and presents an insertion $\mathrm{H}-\mathrm{Au}-\mathrm{H}$ well with a barrier. The reaction dynamics has been studied at zero total angular momentum with quantum WP and QCT methods. The results obtained with the two methods agree very well, validating the use of the QCT method to obtain integral as well as DCSs. The analysis of the results indicates that there are two reaction mechanisms, one direct, dominating at high collision energies, and a second indirect, in which long lived resonances are formed in an insertion $\mathrm{H}-\mathrm{Au}-\mathrm{H}$ well, responsible for the backward peak of the DCS.

The access to the insertion well is dominated by a potential barrier, which is originated by a sequence of conical intersections and avoided crossings with several excited electronic states correlating to the $\mathrm{Au}\left({ }^{2} D,{ }^{2} P\right)+\mathrm{H}_{2}$ asymptotes. The reaction starting in those excited electronic states of gold atoms are exoergic, since their corresponding electronic states are coupled to the ground state through these crossings. The reaction dynamics in the ground as well as in the excited electronic states can be studied experimentally using crossed beams experiments, as in similar studies performed for other transition metals. ${ }^{30-33}$ In addition, the study of the ground and first excited electronic states of increasing $\mathrm{Au}_{N}$ clusters $(N=2,3,4, \cdots)$, and their reactivity, would also be of great interest to understand the catalytic activity of gold clusters and nanostructures. Work in these directions is now in progress.

\section{ACKNOWLEDGMENTS}

This work has been supported by Comunidad Autónoma de Madrid (CAM) under Grant No. S-0505/MAT/0303 and by Ministerio de Ciencia e Innovación under Project Nos. CTQ2007-62898 and CTQ2007-63332.

${ }^{1}$ G. J. Hutchings, J. Catal. 96, 292 (1985).

${ }^{2}$ M. Haruta, N. Yamada, T. Kobayashi, and S. Iijima, J. Catal. 115, 301 (1989).

${ }^{3}$ R. Coquet, K. L. Howard, and D. J. Willock, Chem. Soc. Rev. 37, 2046 (2008).

${ }^{4}$ H. Häkkinen, Chem. Soc. Rev. 37, 1847 (2008).

${ }^{5}$ A. I. Yanson, G. Rubio Bollinger, H. E. van den Brom, N. Agrait, and J. M. van Ruitenbeek, Nature (London) 395, 783 (1998).

${ }^{6}$ H. Ohnishi, Y. Kondo, and K. Takayanagi, Nature (London) 395, 780 (1998).

${ }^{7}$ H. Häkkinen, R. N. Barnett, and U. Landman, J. Phys. Chem. B 103, 8814 (1999).

${ }^{8}$ H. Häkkinen and U. Landman, Phys. Rev. B 62, R2287 (2000).

${ }^{9}$ H. Häkkinen, M. Moseler, and U. Landman, Phys. Rev. Lett. 89, 033401 (2002).

${ }^{10}$ S. Gilb, P. Weis, F. Furche, R. Ahlrichs, and M. M. Kappes, J. Chem. Phys. 116, 4094 (2002).

${ }^{11}$ F. Furche, R. Ahlrichs, P. Weis, C. Jacob, S. Gilb, T. Bierweiler, and M. M. Kappes, J. Chem. Phys. 117, 6982 (2002).

${ }^{12}$ E. M. Fernández, J. M. Soler, I. L. Garzón, and L. C. Balbás, Phys. Rev. B 70, 165403 (2004).

${ }^{13}$ P. Gruene, D. M. Rayner, B. Redlich, A. F. G. van der Meer, J. T. Lyon, G. Maijer, and A. Fielicke, Science 321, 674 (2008).

${ }^{14}$ A. Lechtken, C. Neiss, M. M. Kappes, and D. Schooss, Phys. Chem. Chem. Phys. 11, 4344 (2009). 
${ }^{15}$ R. B. King, Z. Chen, and P. v. R. Schleyer, Inorg. Chem. 43, 4564 (2004).

${ }^{16}$ J. Wang, J. Jellinek, J. Zhao, Z. Chen, R. B. King, and P. v. R. Schleyer, J. Phys. Chem. A 109, 9265 (2005).

${ }^{17}$ S. Bulusu and X. C. Zeng, J. Chem. Phys. 125, 154303 (2006).

${ }^{18}$ M. P. Johansson, D. Sundholm, and J. Vaara, Angew. Chem., Int. Ed. 43, 2678 (2004)

${ }^{19}$ H. Tanaka, S. Neukermans, E. Janssens, R. E. Silverans, and P. Lievens, J. Am. Chem. Soc. 125, 2862 (2003).

${ }^{20}$ C. S. Wannere, C. Corminboeuf, Z.-X. Wang, M. D. Wodrich, R. B. King, and P. v. R. Schleyer, J. Am. Chem. Soc. 127, 5701 (2005).

${ }^{21}$ C. Lemire, R. Meyer, S. Shaikhutdinov, and H.-J. Freund, Angew. Chem., Int. Ed. 43, 118 (2004)

${ }^{22}$ L. Barrio, P. Liu, J. A. Rodriguez, J. M. Campos-Martin, and J. L. G. Fierro, J. Chem. Phys. 125, 164715 (2006).

${ }^{23}$ A. Corma, M. Boronat, S. González, and F. Illas, Chem. Commun. (Cambridge) 3372 (2007).

${ }^{24}$ L. M. Falicov and G. A. Somorjai, Proc. Natl. Acad. Sci. U.S.A. 82, 2207 (1985)

${ }^{25}$ A. Zanchet, A. Dorta-Urra, O. Roncero, F. Flores, C. Tablero, M. Panigua, and A. Aguado, Phys. Chem. Chem. Phys. 11, 10122 (2009).

${ }^{26}$ G.-J. Kang, Z.-X. Chen, Z. Li, and X. He, J. Chem. Phys. 130, 034701 (2009).

${ }^{27}$ R. M. Olson, S. Varganov, M. S. Gordon, H. Metiu, P. Piecuch, S. Cretien, K. Kowalski, S. A. Kucharski, and M. Musial, J. Am. Chem. Soc. 127, 1049 (2005).

${ }^{28}$ Y. C. Choi, W. Y. Kim, H. M. Lee, and K. S. Kim, J. Chem. Theory Comput. 5, 1216 (2009).

${ }^{29}$ S. A. Varganov, R. M. Olson, M. S. Gordon, G. Mills, and H. Metiu, J. Chem. Phys. 120, 5169 (2004)

${ }^{30}$ M. Ishida, R. Yamashiro, Y. Matsumoto, and K. Honma, J. Chem. Phys. 124, 204316 (2006)

${ }^{31}$ R. Yamashiro, Y. Matsumoto, and K. Honma, J. Chem. Phys. 131, 044316 (2009)

${ }^{32}$ R. Z. Hinrichs, P. A. Willis, H. U. Stauffer, J. J. Schroden, and H. Floyd Davis, J. Chem. Phys. 112, 4634 (2000).

${ }^{33}$ J. J. Schroden, H. Floyd Davis, and C. A. Bayse, J. Phys. Chem. A 111 11421 (2007).

${ }^{34}$ K. Balasubramanian and M. Z. Liao, J. Phys. Chem. 92, 361 (1988).

${ }^{35}$ M. Guitou-Guichemerre and G. Chambaud, J. Chem. Phys. 122, 204325 (2005).

${ }^{36}$ L. Andrews, X. Wang, L. Manceron, and K. Balasubramanian, J. Phys. Chem. A 108, 2936 (2004)

${ }^{37}$ D. Figgen, G. Rauhut, M. Dolg, and H. Stoll, Chem. Phys. 311, 227 (2005)

${ }^{38}$ T. H. Dunning, Jr., J. Chem. Phys. 90, 1007 (1989).

${ }^{39}$ MOLPRO, a package of $a b$ initio programs designed by H.-J. Werner and $\mathrm{P}$ J. Knowles, version 2006.1, R. Lindh, F. R. Manby, M. Schütz et al.

${ }^{40}$ H.-J. Werner and P. J. Knowles, J. Chem. Phys. 82, 5053 (1985).

${ }^{41}$ P. J. Knowles and H.-J. Werner, Chem. Phys. Lett. 115, 259 (1985).

${ }^{42}$ H.-J. Werner and P. J. Knowles, J. Chem. Phys. 89, 5803 (1988).

${ }^{43}$ P. J. Knowles and H.-J. Werner, Chem. Phys. Lett. 145, 514 (1988).

${ }^{44}$ E. R. Davidson, J. Comput. Phys. 17, 87 (1975).
${ }^{45}$ P. Schwerdtfeger, J. Reuben Brown, and J. K. Laerdahl, J. Chem. Phys. 113, 7110 (2000).

${ }^{46}$ NIST Chemistry WebBook, NIST Standard Reference Database No. 69, edited by P. J. Linstrom and W. G. Mallard (National Institute of Standards and Technology, Gaithersburg, MD, 2009).

${ }^{47}$ K. P. Huber and G. Herzberg, Molecular Spectra and Molecular Structure, Constants of Diatomic Molecules Vol IV (Van Nostrand, Toronto, 1979).

${ }^{48}$ A. Aguado and M. Paniagua, J. Chem. Phys. 96, 1265 (1992).

${ }^{49}$ A. Aguado, C. Suarez, and M. Paniagua, J. Chem. Phys. 98, 308 (1993).

${ }^{50}$ A. Aguado, C. Tablero, and M. Paniagua, Comput. Phys. Commun. 108, 259 (1998).

${ }^{51}$ R. Rydberg, Z. Phys. 73, 25 (1931).

${ }^{52}$ G. J. Atchity, S. S. Xantheas, and K. Ruedenberg, J. Chem. Phys. 95, $1862(1991)$

${ }^{53}$ A. Aguado, M. Paniagua, M. Lara, and O. Roncero, J. Chem. Phys. 107, 10085 (1997)

${ }^{54}$ M. Lara, A. Aguado, O. Roncero, and M. Paniagua, J. Chem. Phys. 109, 9391 (1998)

${ }^{55}$ T. González-Lezana, A. Aguado, M. Paniagua, and O. Roncero, J. Chem. Phys. 123, 194309 (2005).

${ }^{56}$ Y. Huang, D. J. Kouri, and D. K. Hoffman, J. Chem. Phys. 101, 10493 (1994).

${ }^{57}$ V. A. Mandelshtam and H. S. Taylor, J. Chem. Phys. 103, 2903 (1995).

${ }^{58}$ Y. Huang, S. S. Iyengar, D. J. Kouri, and D. K. Hoffman, J. Chem. Phys. 105, 927 (1996).

${ }^{59}$ G. J. Kroes and D. Neuhauser, J. Chem. Phys. 105, 8690 (1996).

${ }^{60}$ R. Chen and H. Guo, J. Chem. Phys. 105, 3569 (1996).

${ }^{61}$ S. K. Gray and G. G. Balint-Kurti, J. Chem. Phys. 108, 950 (1998).

${ }^{62}$ S. Gómez-Carrasco and O. Roncero, J. Chem. Phys. 125, 054102 (2006).

${ }^{63}$ A. Zanchet, O. Roncero, T. González-Lezana, A. Rodríguez-López, A. Aguado, C. Sanz-Sanz, and S. Gómez-Carrasco, J. Phys. Chem. A 113, 14488 (2009).

${ }^{64}$ M. Karplus, R. N. Porter, and R. D. Sharma, J. Chem. Phys. 43, 3259 (1965).

${ }^{65}$ P. Halvick and J.-C. Rayez, Chem. Phys. 131, 375 (1989).

${ }^{66}$ L. Bonnet and J.-C. Rayez, Chem. Phys. Lett. 277, 183 (1997)

${ }^{67}$ L. Bonnet and J.-C. Rayez, Chem. Phys. Lett. 397, 106 (2004).

${ }^{68}$ L. Bañares, F. J. Aoiz, P. Honvault, B. Bussery-Honvault, and J.-M. Launay, J. Chem. Phys. 118, 565 (2003).

${ }^{69}$ L. Bañares, F. J. Aoiz, P. Honvault, and J.-M. Launay, J. Phys. Chem. 108, 1616 (2004)

${ }^{70}$ A. Zanchet, B. Bussery-Honvault, and P. Honvault, J. Phys. Chem. A 110, 12017 (2006)

${ }^{71}$ A. Zanchet, P. Halvick, J.-C. Rayez, B. Bussery-Honvault, and P. Honvault, J. Chem. Phys. 126, 184308 (2007).

${ }^{72}$ A. Zanchet, P. Halvick, B. Bussery-Honvault, and P. Honvault, J. Chem. Phys. 128, 204301 (2008).

${ }^{73}$ M. Lara, A. Aguado, M. Paniagua, and O. Roncero, J. Chem. Phys. 113, 1781 (2000)

${ }^{74}$ I. R. Elsum and R. G. Gordon, J. Chem. Phys. 76, 3009 (1982).

${ }^{75}$ K. Truhins, R. Marsh, A. J. McCaffery, and T. W. J. Whiteley, J. Chem. Phys. 112, 5281 (2000). 